\title{
RESEARCH
}

Open Access

\section{Survival analysis of extramammary Paget's disease (EMPD) in a tertiary hospital in Taiwan}

Yu-Wei Chang ${ }^{1,2}$, Hsu Ma $a^{1,2}$ and Wen-Chieh Liao ${ }^{1,2,3^{*}}$ (1)

\begin{abstract}
Background: This study aimed to investigate the survival analysis of extramammary Paget's disease (EMPD) in a Taiwanese population and to provide data for comparison with other studies in various locations and racial populations.
\end{abstract}

Methods: We retrospectively analyzed the medical records of 63 patients with EMPD who were surgically treated from 2002 to 2019 at a single institution. The primary endpoint was the 5-year overall survival rate of EMPD, and the secondary endpoint was recurrence-free 5-year survival. Independent variables included patients' demographic data, concurrent malignancy (i.e., non-EMPD-related cancers), tumor size, distant metastasis, and surgery and/or radiation.

Results: Of all the 63 patients, 8 cases were excluded. A total of 43 patients (78.18\%) were male, and 12 were female, with a mean age of 72.67 years (range $44-89$ years). The most common affected anatomic site was the penoscrotal region (22 patients, 40.00\%), followed by the perianal and perineal regions (17 patients, 30.91\%). Among the 55 patients, 41 patients (74.55\%) were diagnosed with at least one underlying disease, whereas the most common underlying disease was cardiovascular disease (30 patients, 54.55\%). The overall survival rate was $80.00 \%$ at 36 months and $65.45 \%$ at the end of follow-up. EMPD with deep dermal invasion was a significant poor prognostic factor of overall survival in cause-specific hazard model (sub-hazard ratio (HR) 5.167, $p=0.0015,95 \%$ confidence interval (Cl) 1.876-14.230). Patients with regional metastasis or distant metastasis had poorer prognosis of 5-year survival (sub-HR 4.513, $p=0.0028, \mathrm{Cl} 1.683-12.103$ ). The limitations of this study include its retrospective nature and sample size.

Conclusions: In our series, EMPD with metastasis and deep dermal invasion was the significant harmful factors in both overall 5-year survival and 5-year recurrence-free survival. The surgical excision is not associated with a low risk of local recurrence or overall survival, and long-term follow-up is still needed.

Keywords: Extramammary Paget disease (EMPD), Survival analysis, Metastasis wide excision

\footnotetext{
* Correspondence: liaowenchieh@gmail.com

'Division of Plastic and Reconstructive Surgery, Department of Surgery,

Taipei Veterans General Hospital, 19F, No.201 Shih-Pai RD Sec 2, Taipei,

Taiwan

${ }^{2}$ School of Medicine, National Yang-Ming University, Taipei, Taiwan

Full list of author information is available at the end of the article
}

(c) The Author(s). 2021 Open Access This article is licensed under a Creative Commons Attribution 4.0 International License, which permits use, sharing, adaptation, distribution and reproduction in any medium or format, as long as you give appropriate credit to the original author(s) and the source, provide a link to the Creative Commons licence, and indicate if changes were made. The images or other third party material in this article are included in the article's Creative Commons licence, unless indicated otherwise in a credit line to the material. If material is not included in the article's Creative Commons licence and your intended use is not permitted by statutory regulation or exceeds the permitted use, you will need to obtain permission directly from the copyright holder. To view a copy of this licence, visit http://creativecommons.org/licenses/by/4.0/. The Creative Commons Public Domain Dedication waiver (http://creativecommons.org/publicdomain/zero/1.0/) applies to the data made available in this article, unless otherwise stated in a credit line to the data. 


\section{Background}

Extramammary Paget's disease (EMPD) is a rare intraepithelial neoplasm that most commonly affects individuals in their 60 s to 80 s $[1,2]$. Given its slow growth and non-specific symptoms, EMPD is easily neglected and results in delayed diagnosis [2-4]. The disease affects sites rich in apocrine glands, including the vulva, scrotum, penis, and perineal and perianal regions and less frequently in the axilla, face, or trunk. High prevalence in Caucasians and predominance in female were reported in Western literature, whereas less frequent occurrence was reported for Asian populations [1-4].

Previous literatures have identified potential factors related to poor prognosis of EMPD; these factors include the dermis invasion, distant metastasis, concurrent malignancy, male gender, and tumor in the perianal anatomic region [2-6]. Karam et al. conducted a survival analysis of white people-predominant population with 2001 EMPD patients in 1973-2007 and concluded the high mortality in invasive EMPD patients with old age, advanced stage, and treatment modality [4].

Different characteristics and manifestations of EMPD in Asian population, including male predominance and low incidence of concurrent internal malignancy, have been identified $[7,8]$. Nevertheless, given the relative rarity of EMPD in Asian population, limited literature reported findings on Taiwanese population [8-11], whereas a similar comprehensive survival analysis in Taiwan is still lacking.

In this study, we presented our 18-year experience of EMPD cases in a single center in Taiwan. We aimed to analyze the demographic characteristic of the disease and identify potential prognostic factors of overall survival and recurrence-free survival in Taiwanese population.

\section{Methods}

\section{Patient selection and inclusion criteria}

This retrospective cohort study was conducted by the plastic surgery department of Taipei Veterans General Hospital, Taiwan. The study was approved by the institutional review board of our hospital. Through the electronic patient record system, in January 2002 to January 2019, patients who received biopsy with final diagnosis of EMPD on pathological reports were included. The 5year survival status was confirmed through electronic patient records. If the survival status cannot be confirmed, phone interview was performed.

\section{Data extraction and selection}

Patient demographic characteristics, including age of diagnosis, gender, concurrent malignancy, anatomic site of lesion, maximal diameter of lesion, and metastasis status, were extracted and recorded. Dermal invasion of the lesion was divided into upper dermis invasion and deep dermal invasion, and the metastasis status was further classified as regional or distant metastasis. The type of treatment was classified into four groups, including surgical excision alone, surgical excision with adjuvant therapy, nonsurgical treatment alone (radiotherapy, chemotherapy, or phototherapy), and without any treatment. In addition to wide local excision, simple or radical vulvectomy in vulva EMPD was included in the excision. Surgical outcomes, including status of excision margin, recurrence, and recurrence-free interval, were also recorded.

\section{Primary and secondary endpoints}

The primary endpoint was the 5-year overall survival rate of EMPD, which was defined as the interval between the date of diagnosis on pathology to the date of death of any cause. The poor prognostic factors of 5-year overall survival were identified. The secondary endpoint was recurrence-free 5-year survival, defined as the interval between the date of diagnosis on pathology to the date of recurrence or death of any cause. The related risk factors of recurrence were also analyzed.

\section{Statistical analysis}

All the data were analyzed by the $\mathrm{SAS}^{\circ} 9.4$ software. Discrete variables were presented in percentages, and the continuous variables were presented as mean and standard deviation. Competing risk analysis with causespecific hazard model was applied to evaluate the variables individually to identify the potential factors of poor prognosis in both 5-year overall survival and recurrence. The proportional hazard assumption of the causespecific hazard model would be tested. Significance was set at $p \leq 0.05$ for each test.

\section{Results}

Between January 2002 and January 2019, 63 patients were diagnosed with EMPD in our hospital. To evaluate the 5-year overall survival status, in addition to the electronic medical record, phone interviews were performed to twelve patients, whereas seven patients were lost to follow-up and one refused the phone interview (Fig. 1). Table 1 lists the demographic characteristics of 55 eligible patients. The mean age diagnosis was 72.67 years (range 44-89 years), with 30 (54.55\%) patients diagnosed at 75 years old or older. The majority of the diagnosed patients were male (43 patients, $78.18 \%$ ), and the most common affected anatomic site was the penoscrotal region (22 patients, $40.00 \%$ ). The second most affected region was the perianal and perineal region (17 patients, $30.91 \%)$. More than half of the patients were diagnosed with a lesion larger than $2 \mathrm{~cm}$ (36 patients, 65.45\%). Among the 55 patients, 41 (74.55\%) were diagnosed with at least one underlying disease, whereas the most 


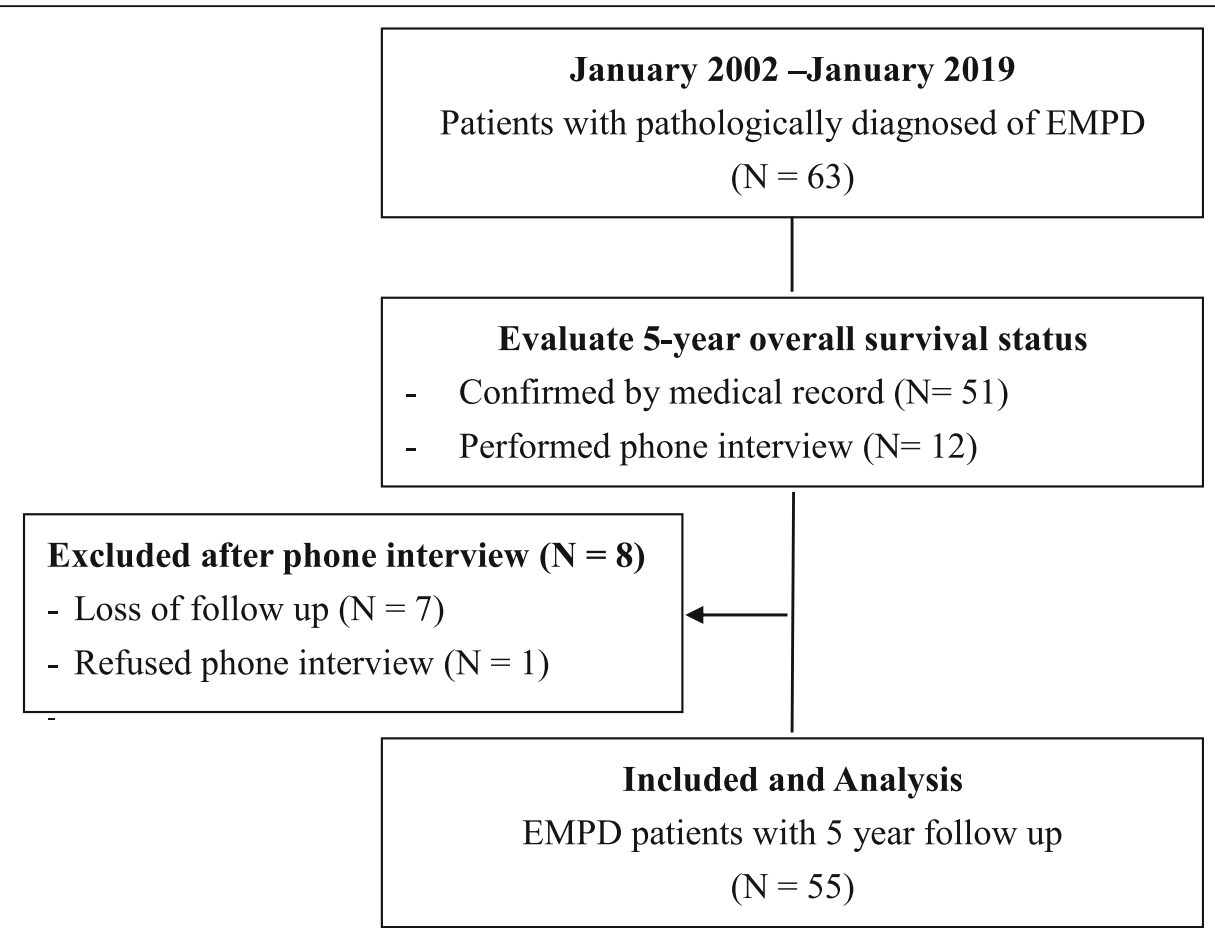

Fig. 1 Data extraction and exclusion and inclusion criteria of our series

common underlying disease was cardiovascular disease (30 patients, $54.55 \%$ ), followed by metabolic or endocrine diseases (15 patients, 27.27\%).

\section{Pathological results and surgical outcomes}

Based on the pathological results of preoperative biopsy, among the 55 eligible patients, 17 had invasive lesions (30.91\%), including 10 lesions with microinvasion of upper dermis (18.18\%) and 7 lesions with deep invasion (12.73\%). Due to lack of the detailed staining results in some patient's electronic record, we analyzed the results of 3 immunohistochemical staining markers among 36 patients, including CK7, CK20, and GCDFP-15 (Fig. 2). Among the 36 immunohistochemically stained EMPD specimen, 33 were positive of CK7 (91.67\%), 8 were positive of CK20 (22.22\%), 5 were positive of GCDFP-15 (13.89\%), and 8 were positive of $\mathrm{Cdx} 2$ (22.22\%) (Table 1). Seven cases indicated metastasis (12.72\%), including three unilateral lymph node metastases $(5.45 \%)$, one bilateral lymph node metastasis $(1.82 \%)$, and three distant metastases $(5.45 \%)$. A total of 47 patients $(85.46 \%)$ received surgical excision of the lesion, including 8 (14.55\%) who received surgical treatment with adjuvant therapy. Four patients $(7.27 \%)$ received radiotherapy or chemotherapy without surgical excision, whereas another four (7.27\%) refused any treatment.

\section{Overall survival rate and prognostic factors}

After diagnosis, the overall survival rate declined over the years (Table 2). The overall survival rate was $80.00 \%$ at 36 months and $65.45 \%$ at the end of follow-up. Cause-specific hazard model of 5-year all-cause mortality was performed (Table 3 ) for each variable. Patients with regional metastasis or distant metastasis had poorer prognosis of 5-year survival compared with patients without metastasis (sub-HR 4.513, $p=0.0028$, CI 1.68312.103). Furthermore, patients with deep dermal invasion had worse prognosis compared with those without dermal invasion (sub-HR 5.167, $p=0.0015$, CI 1.87614.230), whereas no similar harmful effect was noted in the microinvasion of dermis $(p=0.6362)$. No other significant prognostic factor was found among the other variables, including age, anatomic site of lesion, size of lesion, type of treatment, or concurrent malignancy. Proportional hazard assumption was tested, and there was no indication of violating the assumption.

\section{Recurrence rate and 5-year recurrence-free survival}

During the 5-year follow-up, among the fifty-one patients who received any type of treatment, eight patients suffered from recurrence (15.69\%), with a mean recurrence interval of 15.5 months (range 1.3-29.6 months). The recurrence-free survival rate declined more rapidly over the years than the overall survival rate (Table 2). The recurrence-free survival rate was $70.59 \%$ at 36 
Table 1 Demographics and clinical data of 55 study patients with EMPD

\begin{tabular}{|c|c|}
\hline Variable & Patients \\
\hline \multicolumn{2}{|l|}{ Patient characteristics } \\
\hline \multicolumn{2}{|l|}{ Gender (male) } \\
\hline Male & $43(78.18 \%)$ \\
\hline Female & $12(21.82 \%)$ \\
\hline \multicolumn{2}{|l|}{ Age (year) (mean= 72.67, range 44-89) } \\
\hline Age $<65$ years old & $14(25.45 \%)$ \\
\hline Age $=65-74$ years old & $11(20.00 \%)$ \\
\hline Age $=75$ years old or more & $30(54.55 \%)$ \\
\hline \multicolumn{2}{|l|}{ Anatomic site of lesion } \\
\hline Scrotum or penis & $22(40.00 \%)$ \\
\hline Vulva or labia & $7(12.73 \%)$ \\
\hline Perianal or perineal region & $17(30.91 \%)$ \\
\hline Trunk or others & $9(16.36 \%)$ \\
\hline \multicolumn{2}{|l|}{ Types of treatment } \\
\hline Surgical excision only & $39(70.91 \%)$ \\
\hline Surgical excision with adjuvant therapy & $8(14.55 \%)$ \\
\hline Radiotherapy or chemotherapy only & $4(7.27 \%)$ \\
\hline Refused any treatment & $4(7.27 \%)$ \\
\hline Recurrence $(N=51)$ & $8(15.69 \%)$ \\
\hline Concurrent malignancy & $(N=21,38.18 \%)$ \\
\hline Adnexal carcinoma & $3(5.46 \%)$ \\
\hline Internal malignancy & $18(32.73 \%)$ \\
\hline \multicolumn{2}{|l|}{ Underlying diseases } \\
\hline Without any underlying diseases & $14(25.45 \%)$ \\
\hline Cardiovascular diseases & $30(54.55 \%)$ \\
\hline Respiratory diseases & $5(9.09 \%)$ \\
\hline Metabolic or endocrine diseases & $15(27.27 \%)$ \\
\hline Nephrology disease & $3(5.45 \%)$ \\
\hline Gastrointestinal disease & $8(14.55 \%)$ \\
\hline \multicolumn{2}{|l|}{ Pathological parameter } \\
\hline \multicolumn{2}{|l|}{ Size of lesion (length of maximal diameter) } \\
\hline $2 \mathrm{~cm}$ or less than $2 \mathrm{~cm}$ & $19(34.55 \%)$ \\
\hline More than $2 \mathrm{~cm}$ & $36(65.45 \%)$ \\
\hline \multicolumn{2}{|l|}{ Depth of invasion } \\
\hline Intraepithelial & $38(69.09 \%)$ \\
\hline Micro-invasion of upper dermis & $10(18.18 \%)$ \\
\hline Deep invasion & $7(12.73 \%)$ \\
\hline \multicolumn{2}{|l|}{ Immunohistochemical staining $(N=36)$} \\
\hline Positive of CK7 & $33(91.67 \%)$ \\
\hline Positive of CK20 & $8(22.22 \%)$ \\
\hline Positive of GCDFP-15 & $5(13.89 \%)$ \\
\hline Positive of Cdx2 & $8(22.22 \%)$ \\
\hline
\end{tabular}

Table 1 Demographics and clinical data of 55 study patients with EMPD (Continued)

\begin{tabular}{ll}
\hline Variable & Patients \\
\hline Metastasis status $(\mathrm{N}=7,12.72 \%)$ & \\
Unilateral lymph node metastasis & $3(5.45 \%)$ \\
Bilateral lymph node metastasis & $1(1.82 \%)$ \\
Distant metastasis & $3(5.45 \%)$ \\
\hline
\end{tabular}

months and $60.78 \%$ at the end of the follow-up. Competing risk analysis with cause-specific hazard model (Table 4) of recurrence in the 5-year follow-up interval showed a similar outcome as the overall survival. Metastatic disease (sub-hazard ratio 9.103, $p=0.002$, CI 2.249-36.849) and deep dermal invasion (sub-HR 7.836, $p=0.0052$, CI 1.848-33.449) were significant factors leading to poor outcome of recurrence-free survival. No significant association was observed between the margin status and recurrence $(p=0.4338)$. In the subgroup analysis of those with intraepithelial lesion, free-margin status revealed no significant benefit of recurrence-free survival compared with those without free excision margin $(p=0.3998)$. No other significant risk factor of recurrence was found in other variables. Proportional hazard assumption was tested, and there was no indication of violating the assumption.

\section{Concurrent malignancy}

Concurrent or subsequent malignancy was noted in 21 patients $(38.18 \%)$, including 3 patients $(5.45 \%)$ with adnexal carcinoma and 18 patients $(32.73 \%)$ with internal malignancy (Table 1). Among the 18 patients with internal malignancy, 10 were diagnosed with gastrointestinal tract malignancy, 4 with genitourinary tract malignancy, 2 with adenocarcinoma with unknown origin, and 2 with parotid cancer. When analyzed with anatomic site of lesion, among 17 patients with perianal EMPD, 8 patients were diagnosed with gastrointestinal tract malignancy (47.06\%) compared with 2 gastrointestinal tract malignancy in 38 patients with EMPD (5.26\%) in other sites. In the 29 patients with genitourinary EMPD, 3 patients with genitourinary tract malignancy was observed $(10.34 \%)$, whereas a genitourinary tract malignancy was detected in the other 26 EMPD patients (3.85\%). Logistic regression of EMPD anatomic site and internal malignancy revealed the strong association between gastrointestinal malignancy and perianal region EMPD (odds ratio $=16.00, p=0.0015, \mathrm{CI}$ 2.885-88.730), whereas no similar association was noted in genital region EMPD and genitourinary malignancy ( $p$ $=0.3726)($ Table 5).

\section{Discussion}

In the present study, the characteristics of EMPD patients in one single institution were analyzed. As 


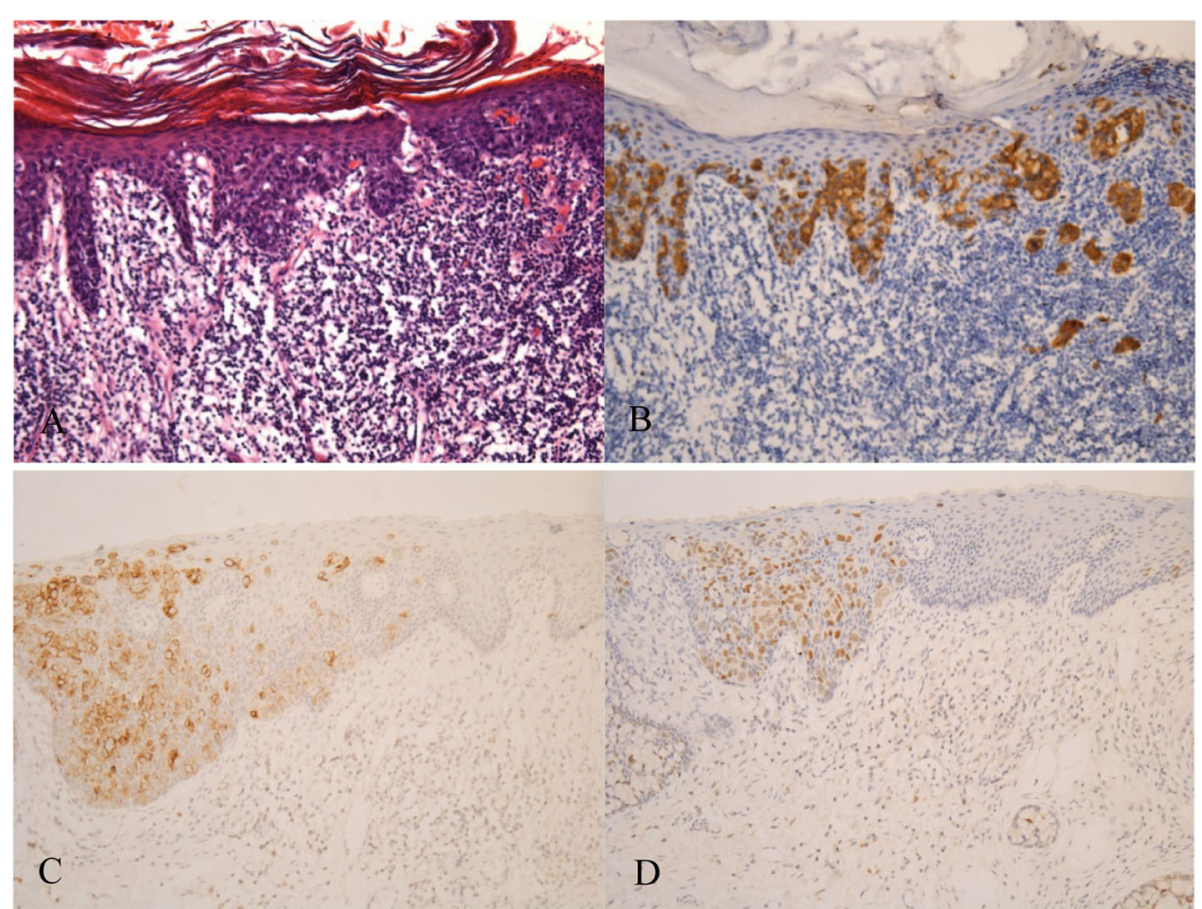

Fig. 2 Images of immunohistochemical staining in EMPD of our series. a Primary EMPD with micro-invasion $\times 10$. b Positive expression of CK7 $\times 10$. c Positive expression of CK20 $\times 10$. $\mathbf{d}$ Positive expression of $\mathrm{Cd} \times 2 \times 10$

revealed in other Asian population-based studies [7, 12], the predominance of male gender in the distribution of EMPD patients was also noted in our cases. The most common affected site was the penoscrotal region $(40 \%)$, similar to the findings of other studies [13-15]. The average size of lesion, the mean age of diagnosis, and rate of metastasis $(12.72 \%)$ were also in compatible range with previous literature $[4,5,7,12]$

Table 2 Five-year overall survival rate and recurrence-free survival rate

\begin{tabular}{lcl}
\hline Years after diagnosis & Number or survival & Rate \\
5-year overall survival rate $(N=55)$ & \\
1st year & 51 & $92.73 \%$ \\
2nd year & 47 & $85.45 \%$ \\
3rd year & 44 & $80.00 \%$ \\
4th year & 41 & $74.55 \%$ \\
5th year & 36 & $65.45 \%$ \\
Years after diagnosis & Number of recurrence-free & Recurrence-free \\
& survival & survival rate \\
5-year recurrence-free survival rate ( $N=51)$ & \\
1st year & 46 & $90.20 \%$ \\
2nd year & 38 & $74.51 \%$ \\
3rd year & 36 & $70.59 \%$ \\
4 th year & 34 & $66.67 \%$ \\
5th year & 31 & $60.78 \%$ \\
\hline
\end{tabular}

The overall survival rates in our study were $80.00 \%$ (36-month follow-up) and 65.45\% (60-month follow-up) (Table 2), which were compatible with those of previous male-predominant or Asian-predominant study [12, 13]. Previous studies had identified several potential risk factors of poor prognosis of EMPD, including the level of tumor invasion, lymph node metastases, elevated CEA, perianal lesion, old age, and male gender $[5,12,13,16]$. In our study, based on the results of cause-specific hazard model (Table 3), metastatic diseases and deep dermal invasion were identified as significant harmful factors of the overall 5-year survival, showing similar outcomes with two population-based studies and previous reviews $[6,12,17]$. The relationship between survival and microinvasive disease remains controversial, whereas deeply invasive EMPD was linked to poorer prognosis than the non-invasive counterpart [12, 17]. The association between prognosis and site of lesion had been reported, suggesting that anorectal EMPD has a statistically significantly decreased mean disease-specific survival compared with those without anorectal involvement [4]. However, no significant difference in overall survival was observed between the different groups of lesion site in our study (Table 3).

The 5-year recurrence rate (15.69\%) and the mean recurrence interval (15.5 months after diagnosed) in our study were similar to those of other EMPD studies that treated patients with wide local excision $[14,15,18]$. 
Table 3 Competing risk analysis of 5-year mortality

\begin{tabular}{|c|c|c|c|c|}
\hline Variate & Mortality rate & Sub-HR & $95 \% \mathrm{Cl}$ & $p$-value \\
\hline \multicolumn{5}{|l|}{ Gender } \\
\hline Female & $16.67 \%$ & Reference & Reference & Reference \\
\hline Male & $39.53 \%$ & 2.737 & $0.632-11.859$ & 0.1784 \\
\hline \multicolumn{5}{|l|}{ Age } \\
\hline Age less than 75 years old & $24.00 \%$ & Reference & Reference & Reference \\
\hline Age $=75$ years old or more & $43.33 \%$ & 2.127 & $0.808-5.600$ & 0.1265 \\
\hline \multicolumn{5}{|l|}{ Lesion site } \\
\hline Genital region or others & $26.32 \%$ & Reference & Reference & Reference \\
\hline Perianal or perineal region & $52.94 \%$ & 2.338 & $0.948-5.765$ & 0.0651 \\
\hline \multicolumn{5}{|l|}{ Size of lesion } \\
\hline Lesion $=2 \mathrm{~cm}$ or less & $26.32 \%$ & Reference & Reference & Reference \\
\hline Lesion lager than $2 \mathrm{~cm}$ & $38.89 \%$ & 1.624 & $0.584-4.514$ & 0.3527 \\
\hline \multicolumn{5}{|l|}{ Invasion of dermis } \\
\hline No dermal invasion & $28.95 \%$ & Reference & Reference & Reference \\
\hline Micro-invasion & $20.00 \%$ & 0.695 & $0.154-3.137$ & 0.6362 \\
\hline Deep dermal invasion & $85.71 \%$ & 5.167 & $1.876-14.230$ & 0.0015 \\
\hline \multicolumn{5}{|l|}{ Metastasis status } \\
\hline Without metastasis & $27.08 \%$ & Reference & Reference & Reference \\
\hline Metastatic diseases & $85.71 \%$ & 4.513 & $1.683-12.103$ & 0.0028 \\
\hline \multicolumn{5}{|l|}{ Recurrence $(N=51)$} \\
\hline No recurrence & $27.91 \%$ & Reference & Reference & Reference \\
\hline With recurrence & $62.50 \%$ & 2.587 & $0.907-7.382$ & 0.0756 \\
\hline \multicolumn{5}{|l|}{ Concurrent malignancy } \\
\hline No concurrent malignancy & $29.41 \%$ & Reference & Reference & Reference \\
\hline Adnexal carcinoma & $33.33 \%$ & 1.180 & $0.151-9.225$ & 0.8744 \\
\hline Internal malignancy & $44.44 \%$ & 1.595 & $0.629-4.044$ & 0.3249 \\
\hline \multicolumn{5}{|l|}{ Types of treatment } \\
\hline With surgical excision & $31.91 \%$ & Reference & Reference & Reference \\
\hline Without surgical excision & $50.00 \%$ & 1.642 & $0.544-4.950$ & 0.3787 \\
\hline \multicolumn{5}{|l|}{ Margin status $(N=47)$} \\
\hline Margin not free & $33.33 \%$ & Reference & Reference & Reference \\
\hline Margin free & $31.03 \%$ & 0.911 & $0.324-2.561$ & 0.8596 \\
\hline \multicolumn{5}{|l|}{ Intraepithelial lesion ( $N=31$ ) } \\
\hline Margin not free & $37.50 \%$ & Reference & Reference & Reference \\
\hline Margin free & $21.74 \%$ & 0.528 & $0.126-2.211$ & 0.3822 \\
\hline
\end{tabular}

Abbreviation: $\mathrm{Cl}$ confidence interval, Sub-HR sub-hazard ratio

The recurrence-free survival rate was $70.59 \%$ at 36 month follow-up and $60.78 \%$ at 60 -month follow up (Table 2), consistent with those of other wide local excision studies $[15,18]$. Based on the results of competing risk analysis (Table 4), metastatic diseases and deep dermal invasion were identified as potential risk factors of recurrence. The results of our study coincided with that of a previous study [10], whereas another populationbased study reported no relationship between dermal invasion and local recurrence [7]. Previous literature observed a strong association between margin status and recurrence risk [15], whereas in our study (Table 4) no similar significant association was found. In the subgroup analysis of those with intraepithelial lesion, freemargin status revealed no improvement in recurrencefree survival compared with those without free excision margin $(p=0.3998)$, which was in conflict with previous literature [19]. 
Table 4 Competing risk analysis of recurrence $(N=51)$

\begin{tabular}{|c|c|c|c|c|}
\hline Variate & Recurrence rate & Sub-HR & $95 \% \mathrm{Cl}$ & $p$-value \\
\hline \multicolumn{5}{|l|}{ Gender } \\
\hline Female $(N=12)$ & $16.67 \%$ & Reference & Reference & Reference \\
\hline Male $(N=39)$ & $15.38 \%$ & 0.884 & $0.178-4.381$ & 0.8799 \\
\hline \multicolumn{5}{|l|}{ Age } \\
\hline Age less than 75 years old $(N=25)$ & $20.00 \%$ & Reference & Reference & Reference \\
\hline Age $=75$ years old or more $(N=26)$ & $11.54 \%$ & 0.673 & $0.161-2.818$ & 0.5878 \\
\hline \multicolumn{5}{|l|}{ Lesion site } \\
\hline Genital region or other $(N=36)$ & $16.67 \%$ & Reference & Reference & Reference \\
\hline Perianal or perineal $(N=15)$ & $13.33 \%$ & 0.835 & $0.169-4.138$ & 0.8253 \\
\hline \multicolumn{5}{|l|}{ Size of lesion } \\
\hline Lesion $=2 \mathrm{~cm}$ or less $(N=16)$ & $6.25 \%$ & Reference & Reference & Reference \\
\hline Lesion lager than $2 \mathrm{~cm}(\mathrm{~N}=35)$ & $20.00 \%$ & 3.743 & $0.460-30.454$ & 0.2171 \\
\hline \multicolumn{5}{|l|}{ Invasion of dermis } \\
\hline No dermal invasion $(N=45)$ & $11.11 \%$ & Reference & Reference & Reference \\
\hline Deep dermal invasion $(N=6)$ & $50.00 \%$ & 7.863 & $1.848-33.449$ & 0.0052 \\
\hline \multicolumn{5}{|l|}{ Metastasis } \\
\hline Without metastasis $(N=44)$ & $9.09 \%$ & Reference & Reference & Reference \\
\hline With any metastasis $(N=7)$ & $57.14 \%$ & 9.103 & $2.249-36.849$ & 0.0020 \\
\hline \multicolumn{5}{|l|}{ Concurrent malignancy } \\
\hline No concurrent malignancy $(N=32)$ & $12.50 \%$ & Reference & Reference & Reference \\
\hline Adnexal or Internal malignancy $(N=19)$ & $21.05 \%$ & 1.955 & $0.488-7.828$ & 0.3436 \\
\hline \multicolumn{5}{|l|}{ Excision margin status $(N=47)$} \\
\hline Margin not free $(N=18)$ & $22.22 \%$ & Reference & Reference & Reference \\
\hline Margin free $(N=29)$ & $13.79 \%$ & 0.575 & $0.144-2.300$ & 0.4338 \\
\hline \multicolumn{5}{|l|}{ Intraepithelial lesion ( $N=31)$} \\
\hline Margin not free $(N=8)$ & $25.00 \%$ & Reference & Reference & Reference \\
\hline Margin free $(N=23)$ & $13.04 \%$ & 0.463 & $0.077-2.779$ & 0.3998 \\
\hline
\end{tabular}

Abbreviation: $\mathrm{Cl}$ confidence interval, Sub-HR sub-hazard ratio

The rates of concurrent malignancy (38.18\%), adnexal carcinoma (5.45\%), and internal malignancy (32.73\%) in our study were in compatible range with previous reviews $[2,5,15,20]$. Several Asian population-based studies revealed a low concurrent internal malignancy rate in Asian EMPD patients [7, 12, 14] which is in contrast with the result of our study. The potential relationship between the anatomic site of EMPD lesion and internal malignancy was proposed in another study [20]. We determined the perianal EMPD as a significant risk factor of gastrointestinal malignancy (odds ratio $=16.00, p=0.0015$, CI 2.885-88.730), whereas no similar association was observed between the genital region EMPD and genitourinary malignancy $(p=0.3726)$ (Table 5).

Our study had several limitations. First, all the data were retrospectively extracted from the electronic patient record system, which may lead to potential bias in data extraction or misinterpretation. Inadequate description of pathology reports and outpatient department follow-up may also lead to underestimation of the actual rate of dermis invasion and recurrence. In addition,

Table 5 Logistic regression analysis of EMPD and internal malignancy

\begin{tabular}{|c|c|c|c|}
\hline Variate & Odds ratio & 95\% confidence interval & $p$-value \\
\hline Perianal EMPD and gastrointestinal malignancy & 16.000 & $2.885-88.730$ & 0.0015 \\
\hline Genital region EMPD and genitourinary malignancy & 2.884 & $0.281-29.609$ & 0.3726 \\
\hline
\end{tabular}


given the long follow-up period of up to 5 years, phone interview was performed as an alternative way of evaluation, in which only limited information can be accessed. Finally, with the rarity of EMPD in Asian population, the present single-center study included 55 illegible patients. A multicenter, larger sample size study in Taiwanese population is still needed for further evaluation.

To the best of our knowledge, this research is the first study in the English language literature about the comprehensive survival analysis of EMPD in Taiwan population. Our report also identified similar disease characteristics and prognostic factors in Taiwan population, similar to other Asian population-based studies, and their differences.

\section{Conclusion}

EMPD is commonly observed among aged people. The presence of metastatic EMPD and deep dermal invasion are significant harmful factors of the overall 5 -year survival and 5-year recurrence-free survival. In most cases, EMPD is not associated with cancer, whereas perianal EMPD is accompanied with a high risk of gastrointestinal malignancy. Regardless of treatment method, longterm follow-up is recommended.

\section{Abbreviations}

EMPD: Extramammary Paget's disease; sub-HR: Sub-hazard ratio

\section{Acknowledgements}

The authors would like to thank Y. L. LIN HUNG TAI Education and Culture Charity Trust and Y. L. LIN HUNG TAI Education Foundation for kindly funding the article-processing charge of this article and the Enago academy (www.enago.tw) for their expertise and assistance in professional language editing of the manuscript.

\section{Authors' contributions}

WL collected the data and assisted with manuscript editing. $Y C$ analyzed the data and wrote the manuscript. HM designed and supervised the study. All authors participated in final revision and approved the manuscript.

\section{Funding}

Not applicable.

\section{Availability of data and materials}

The datasets used and/or analyzed during the current study are available from the corresponding author on reasonable request.

\section{Declarations}

\section{Ethics approval and consent to participate}

This study was approved and supervised by the institutional review board of Taipei Veterans General Hospital (Approval number:2020-03-021CC).

\section{Consent for publication}

Not applicable.

\section{Competing interests}

The authors declare that they have no competing interests.

\section{Author details}

'Division of Plastic and Reconstructive Surgery, Department of Surgery, Taipei Veterans General Hospital, 19F, No.201 Shih-Pai RD Sec 2, Taipei, Taiwan. ${ }^{2}$ School of Medicine, National Yang-Ming University, Taipei, Taiwan.
${ }^{3}$ Institute of Environmental and Occupational Health Sciences, School of Medicine, National Yang Ming Chiao Tung University, Taipei, Taiwan.

Received: 13 November 2020 Accepted: 3 April 2021

Published online: 12 April 2021

References

1. Adashek JJ, Leonard A, Nealon SW, Krishnan A, Mosiello GC, Dhillon J, et al. Extramammary Paget's disease: what do we know and how do we treat? Can J Urol. 2019;26(6):10012-21.

2. Asel M, LeBoeuf NR. Extramammary Paget's disease. Hematol Oncol Clin North Am. 2019;33(1):73-85. https://doi.org/10.1016/j.hoc.2018.09.003.

3. Merritt BG, Degesys CA, Brodland DG. Extramammary Paget disease. Dermatol Clin. 2019;37(3):261-7. https://doi.org/10.1016/j.det.2019.02.002.

4. Karam A, Dorigo O. Treatment outcomes in a large cohort of patients with invasive Extramammary Paget's disease. Gynecol Oncol. 2012;125(2):346-51. https://doi.org/10.1016/j.ygyno.2012.01.032.

5. Yao H, Xie M, Fu S, Guo J, Peng Y, Cai Z, et al. Survival analysis of patients with invasive extramammary Paget disease: implications of anatomic sites. BMC Cancer. 2018;18(1):403. https://doi.org/10.1186/s12885-018-4257-1.

6. Cohen JM, Granter SR, Werchniak AE. Risk stratification in extramammary Paget disease. Clin Exp Dermatol. 2015:40(5):473-8. https://doi.org/10.1111/ced.12690.

7. Chan JY, Li GK, Chung JH, Chow VL. Extramammary Paget's disease: 20 years of experience in Chinese population. Int J Surg Oncol. 2012;2012:416418.

8. Cheng PS, Lu CL, Cheng CL, Lai FJ. Significant male predisposition in extramammary Paget disease: a nationwide population-based study in Taiwan. Br J Dermatol. 2014;171(1):191-3. https://doi.org/10.1111/bjd.12851.

9. Wang YC, Li AF, Yang SH, Ma HH, Liang WY. Perianal Paget's disease: the 15year-experience of a single institution in Taiwan. Gastroenterol Res Pract. 2019;2019:2603279.

10. Lai YL, Yang WG, Tsay PK, Swei H, Chuang SS, Wen CJ. Penoscrotal extramammary Paget's disease: a review of 33 cases in a 20-year experience. Plast Reconstr Surg. 2003;112(4):1017-23. https://doi.org/10.1097/01.PRS.0000076193.67701.6A

11. Chiu CS, Yang $\mathrm{CH}$, Chen $\mathrm{CH}$. Extramammary Paget's disease of the unilateral axilla: a review of seven cases in a 20-year experience. Int J Dermatol. 2011 ; 50(2):157-60. https://doi.org/10.1111/j.1365-4632.2010.04604.x.

12. Ito $Y$, Igawa S, Ohishi $Y$, Uehara J, Yamamoto Al, lizuka H. Prognostic indicators in 35 patients with extramammary Paget's disease. Dermatol Surg. 2012;38(12):1938-44. https://doi.org/10.1111/j.1524-4725.2012.02584.x.

13. Herrel LA, Weiss AD, Goodman M, Johnson TV, Osunkoya AO, Delman KA, et al. Extramammary Paget's disease in males: survival outcomes in 495 patients. Ann Surg Oncol. 2015;22(5):1625-30. https://doi.org/10.1245/s1 0434-014-4139-y.

14. Chiu TW, Wong PS, Ahmed K, Lam SC, Ying SY, Burd A. Extramammary Paget's disease in Chinese males: a 21-year experience. World J Surg. 2007; 31(10):1941-6. https://doi.org/10.1007/s00268-007-9189-x.

15. Long B, Schmitt AR, Weaver AL, McGree M, Bakkum-Gamez JN, Brewer J, et al. A matter of margins: surgical and pathologic risk factors for recurrence in extramammary Paget's disease. Gynecol Oncol. 2017;147(2):358-63. https://doi.org/10.1016/j.ygyno.2017.09.008.

16. Leong JY, Chung PH. A primer on extramammary Paget's disease for the urologist. Transl Androl Urol. 2020;9(1):93-105. https://doi.org/10.21037/tau.2 019.07.14.

17. Hatta N, Yamada M, Hirano T, Fujimoto A, Morita R. Extramammary Paget's disease: treatment, prognostic factors and outcome in 76 patients. $\mathrm{Br} J$ Dermatol. 2008;158(2):313-8. https://doi.org/10.1111/j.1365-2133.2007.08314.x.

18. Lee KY, Roh MR, Chung WG, Chung KY. Comparison of mohs micrographic surgery and wide excision for extramammary Paget's disease: Korean experience. Dermatol Surg. 2009;35(1):34-40. https://doi.org/10.1111/j.1524-4 725.2008.34380.x.

19. Hegarty PK, Suh J, Fisher MB, Taylor J, Nguyen TH, Ivan D, et al. Penoscrotal extramammary Paget's disease: the university of Texas M. D. Anderson cancer center contemporary experience. J Urol. 2011;186(1):97-102. https:// doi.org/10.1016/j.juro.2011.02.2685

20. Lam C, Funaro D. Extramammary Paget's disease: summary of current knowledge. Dermatol Clin. 2010;28(4):807-26. https://doi.org/10.1016/j.det.2010.08.002

\section{Publisher's Note}

Springer Nature remains neutral with regard to jurisdictional claims in published maps and institutional affiliations. 